\title{
A Case of Adams-Oliver Syndrome
}

\author{
Jong-Keun Seo, M.D., Ju-Hyun Kang, M.D., Hyun-Jae Lee, M.D., Deborah Lee, M.D., \\ Ho-Suk Sung, M.D., Seon-Wook Hwang, M.D.
}

Department of Dermatology, Busan Paik Hospital, College of Medicine, Inje University, Busan, Korea

\begin{abstract}
Adams-Oliver syndrome (AOS) is a congenital condition characterized by aplasia cutis congenita, transverse limb defects, and cutis marmorata telangiectatica. AOS can also be associated with extensive lethal anomalies of internal organs, including the central nervous, cardiopulmonary, gastrourointestinal, and genitourinary systems. Generally, the more severe these interrelated anomalies are, the poorer the prognosis becomes. In the relevant literature on this topic, it is somewhat unclear as to whether the prognosis of AOS without lethal anomalies alters the lifespan. We report a case of AOS with typical skin defects only, and no internal organ anomalies. (Ann Dermatol 22(1) 96 98, 2010)
\end{abstract}

\section{-Keywords-}

Adams-Oliver syndrome, Aplasia cutis congenita, Cutis marmorata telangiectatica

\section{INTRODUCTION}

Adams-Oliver syndrome (AOS) is a rare congenital anomaly complex characterized by aplasia cutis congenita in the vertex area, terminal transverse defects of the limbs, and cutis marmorata telangiectatica congenital (CMTC). As this syndrome has severe forms of expression, including central nervous system abnormalities, cardiovascular disease, and gastrointestinal malformations, patients with this syndrome usually die within several weeks. To the best of our knowledge, no report yet has discussed the lifespan of AOS patients who do not evidence lethal anomalies.

Received March 19, 2009, Revised July 2, 2009, Accepted for publication July 27, 2009

Reprint request to: Deborah Lee, M.D., Department of Dermatology, Busan Paik Hospital, College of Medicine, Inje University, 633-165, Gaegeum-dong, Busanjin-gu, Busan 614-735, Korea. Tel: 82-51-8906135, Fax: 82-51-897-6391, E-mail: paikderma@hanmail.net

\section{CASE REPORT}

A 21-year-old man presented with a hairless atrophic patch (Fig. 1), CMTC on the dorsum of both hands, shortening of the fingers and toes, interdigital webs of the 2nd and 3rd toes, and total absence of the right 3rd toenail (Fig. 2). He was born at term and presented with small hairless patches over his scalp and total absence of the right 3 rd toenail. The patient reported no history of maternal drug intake, infection, or radiation exposure during the pregnancy and no familial history of AOS, mental retardation, or CNS abnormalities. The scalp had a well-demarcated $10 \times 12 \mathrm{~cm}$ hairless atrophic patch at its vertex. Histopathological examination of the scalp lesion revealed rete ridge flattening, collagen bundle thickening in the dermis, and the loss of appendages (Fig. 3). The skull x-ray showed a thinning of the bone underlying the hairless atrophic patch. No abnormalities were noted on brain computed tomography $(\mathrm{CT})$ or on chest $\mathrm{x}$-ray. The

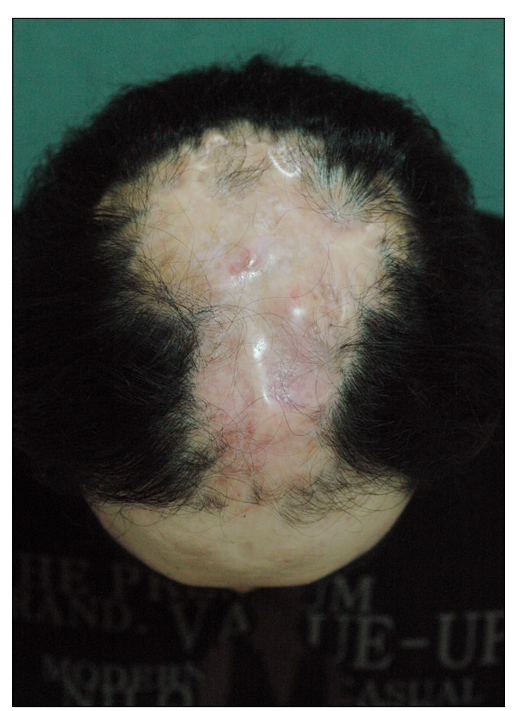

Fig. 1. Alopecic scar of aplasia cutis $(10 \times 12 \mathrm{~cm})$ at the vertex. 

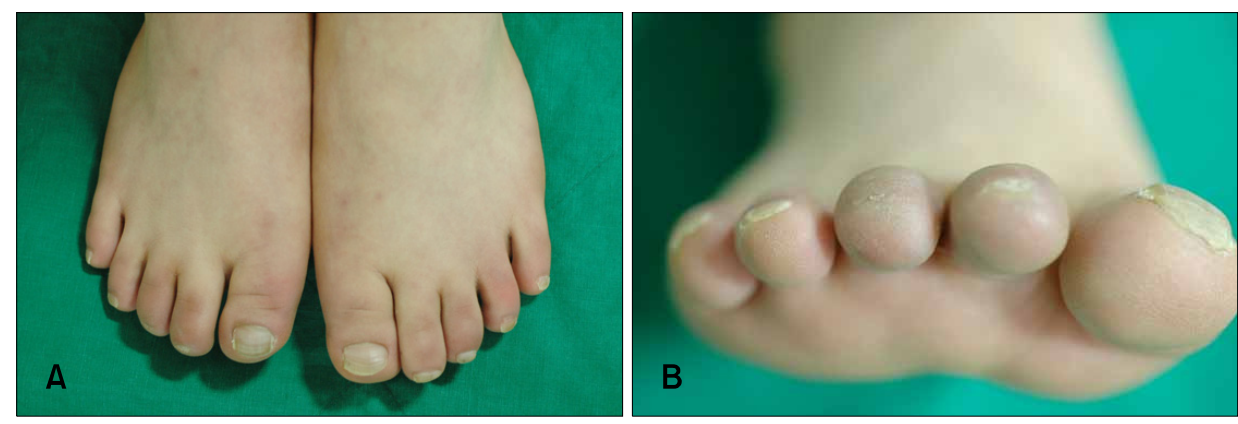

Fig. 2. (A) Brachydactyly and cutis marmorata telangiectatica on the dorsa of both feet and (B) the absence of the right 3 rd toenail.

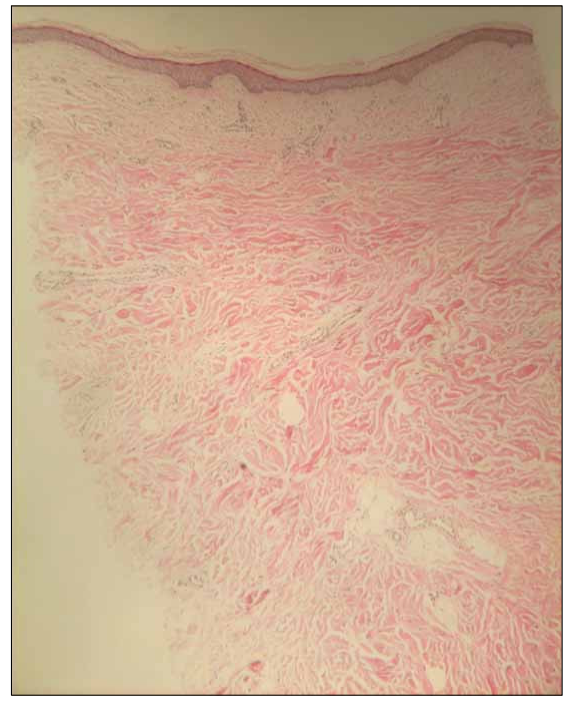

Fig. 3. Histopathologically, the hairless atrophic patch on the scalp showed rete ridge flattening, collagen bundle thickening, and loss of appendage $(\mathrm{H} \& \mathrm{E}, \times 40)$.

combination of aplasia cutis congenita and skeletal defects of the extremities resulted in a diagnosis of AOS. Gastrointestinal and urologic system examinations were recommended to the patient, but he refused to undergo them because he had already survived for more than 20 years with AOS.

\section{DISCUSSION}

AOS is a congenital disease characterized by aplasia cutis congenita, transverse limb defects, and $\mathrm{CMTC}^{1,2}$. It was initially described in 1945 by Adams and Oliver ${ }^{1}$. The disease largely has an autosomal dominant mode of inheritance, although autosomal recessive and sporadic modes often occur as well ${ }^{3,4}$. Our patient had no family history of congenital scalp or limb defects. Therefore, this case was likely a sporadic one.

The limb defects, the most common feature of this disease, are usually asymmetric. The lower limbs are more susceptible than the upper limbs. The range of involve- ment includes brachydactyly, syndactyly, agenesis of fingernails or toenails, loss of the terminal phalanges, or even more severe defects such as the complete absence of a finger, toe, hand, foot, or limb $b^{5,6}$. Our patient had interdigital webs of the 2 nd and $3 r d$ toes and absence of the $3 r d$ toenail on the right. Scalp defects are the second most frequent finding in $\mathrm{AOS}^{4}$. The scalp lesions occasionally extend through the skull deformities. Our patient exhibited only a scalp defect and skull thinning. The lesion was confirmed as aplasia cutis congenita upon histopathological examination. Other associated defects include CMTC, central nervous system and cardiovascular malformations, accessory nipples, microphthalmia, and cleft $\operatorname{lip}^{7,8}$. Among these defects, our patient exhibited only CMTC.

Although the exact pathogenesis of AOS remains unknown, vascular impairment during embryogenesis has been proposed as a possible mechanism. In order to identify the genetic cause of AOS, Verdyck et al. ${ }^{9}$ evaluated a family with 10 affected individuals over four generations. However, they failed to find any disease- causing mutations.

Various expressions of AOS have been reported. This report shows that AOS without major organ abnormalities does not necessarily alter the normal lifespan. Additionally, if a newborn presents with aplasia cutis congenita and limb defects, dermatologists should consider AOS by conducting an evaluation of the central nervous system and looking for cardiovascular, gastrointestinal, and genitourinary malformations.

\section{REFERENCES}

1. Adams FH, Oliver CP. Hereditary deformities in man: due to arrested development. J Hered 1945;36:3-7.

2. Mempel M, Abeck D, Lange I, Strom K, Caliebe A, Beham $A$, et al. The wide spectrum of clinical expression in AdamsOliver syndrome: a report of two cases. $\mathrm{Br} J$ Dermatol 1999;140:1157-1160.

3. Becker R, Kunze J, Horn D, Gasiorek-Wiens A, Entezami M, Rossi $R$, et al. Autosomal recessive type of Adams-Oliver 
syndrome: prenatal diagnosis. Ultrasound Obstet Gynecol 2002;20:506-510.

4. Narang T, Kanwar AJ, Dogra S. Adams-Oliver syndrome: a sporadic occurrence with minimal disease expression. Pediatr Dermatol 2008;25:115-116.

5. Dadzie OE, Tyszczuk L, Holder SE, Teixeira F, Charakida A, Scarisbrick J, et al. Adams-Oliver syndrome with widespread CMTC and fatal pulmonary vascular disease. Pediatr Dermatol 2007;24:651-653.

6. Sankhyan N, Kaushal RK, Jaswal RS. Adams-Oliver syndrome: a case with complete expression. J Dermatol 2006;
33:435-436

7. McGoey RR, Lacassie Y. Adams-Oliver syndrome in siblings with central nervous system findings, epilepsy, and developmental delay: refining the features of a severe autosomal recessive variant. Am J Med Genet A 2008;146A:488-491.

8. Jun SY, Khang SK, Park SH. An autopsy case of AdamsOliver syndrome. J Korean Med Sci 2000;15:482-484.

9. Verdyck P, Blaumeiser B, Holder-Espinasse M, Van Hul W, Wuyts W. Adams-Oliver syndrome: clinical description of a four-generation family and exclusion of five candidate genes. Clin Genet 2006;69:86-92. 